Jan LAZAR

Université d'Opole / POLOGNE

Andrzej NAPIERALSKI

Université de Lodz / POLOGNE

Jean-François SABLAYROLLES

Université Paris 13, HTL UMR 7597

http://dx.doi.org/10.18778/8088-785-5.04

\title{
Le selfie et ses différentes formes : l'amour de soi et le corps en morceaux
}

\section{Résumé}

S’occuper de son corps, par l'exercice ou par des soins, n'est pas nouveau, mais une place de plus en plus importante y est accordée de nos jours, comme le montrent la multiplication des centres de musculation et les multiples publicités pour les produits cosmétiques. Une autre forme de cet intérêt pour soi-même s'est développée récemment avec l'essor des selfies (egoportraits en français québécois), et cela sur toute la planète ou peu s'en faut. Mais les selfies se sont vite diversifiés et ce sont précisément ces innovations et leurs dénominations néologiques, essentiellement anglo-américaines, que nous nous proposons d'étudier en comparant le français, le polonais et le tchèque. Il semble qu'on ait d'abord photographié et diffusé sur les réseaux sociaux certaines parties de son anatomie, puis qu'on se soit pris en photo dans certaines attitudes ou dans certaines occupations. Des dénominations ont aussi été inventées pour le nombre de personnes figurant sur le cliché en plus de soi-même. Parallèlement, des évolutions se sont manifestées dans la dénomination du selfie, qui, outre cette forme complète, est représentée par les fractolexèmes -elfie, -lfie, -fie, et même seulement -ie. Ce qui peut donner lieu à l'apparition de dénominations opaques sans contexte et sans photo (helfie) et aussi de synonymes (legsie, lelfie). On constate aussi la création d'homonymes (relfie). Des articles de la presse généraliste nous ont fait découvrir ces nouvelles 
formes, puis des recherches de celles-ci sur l'internet ont permis d'en trouver d'autres. Toutes ne sont néanmoins pas aussi utilisées les unes que les autres et nous prenons en compte leur circulation dans la presse généraliste de nos trois langues ainsi que sur les sites répertoriés par des moteurs de recherche.

Mots-clés : néologisme, emprunt, facebook, selfie, réseaux sociaux

\section{Summary}

Taking care of one's body by exercising or by use of a body care is nothing new, but an increasingly important place is given to it nowadays, as it is shown by the enormous growth of sport centres and numerous advertisements for cosmetic products. Another form of this interest in oneself has recently developed with the increase in selfies (egoportraits in Quebec French) all over the world or almost. Nonetheless, the selfies have rapidly become varied and these innovations and their neological denominations, essentially Anglo-American ones, are exactly what we propose to study in comparison with the French, the Polish and the Czech. The common press articles allowed us to discover these new forms and the following research of these on the Internet have facilitated the way to find others. However, not all of them are used and we take into account their occurence in the general press of the three languages as well as on sites listed by search engines.

Keywords: neologism, borrowing, facebook, selfie, social media

\section{Introduction}

S’occuper de son corps, par l'exercice ou par des soins de beauté, n'est pas nouveau, comme l'atteste l'Antiquité grecque et romaine, mais une place de plus en plus importante y est accordée dans la société contemporaine. La multiplication des centres de fitness et les publicités pour les produits cosmétiques en sont des manifestations évidentes. Une autre forme de cet intérêt pour soi-même s'est développée avec l'essor des selfies (egoportraits en français québécois). Outre que cette pratique est omniprésente au point que des réglementations ont dû être édictées pour l'interdire dans certains lieux et certaines circonstances du fait de la gêne causée à autrui ou de risque pour soi ${ }^{1}$, elle s'est diversifiée dans ses

1 En France les selfies sont autorisés dans les musées mais les cannes à selfie sont interdites dans beaucoup d'entre eux. En janvier 2016, on estime à 27 le nombre de morts imputables à la prise de selfies, dont la moitié en Inde. 
formes. Et ce sont précisément ces innovations et leurs dénominations néologiques que nous nous proposons de recenser (sans prétendre atteindre l'exhaustivité) et d'étudier en français, polonais et tchèque.

Sans qu'il soit possible d'établir une chronologie précise, il semble qu'on ait d'abord photographié et diffusé sur les réseaux sociaux certaines parties de son anatomie, vêtues ou non, puis qu'on se soit pris en photo dans certaines attitudes, occupations ou états. Des dénominations ont aussi été inventées pour le nombre et la qualité des personnes figurant sur le cliché en plus de soi-même. Parallèlement, des évolutions se sont manifestées dans la dénomination du selfie, qui, outre cette forme, est représentée par les fractolexèmes -elfie, -lfie, -fie, et même seulement -ie.

C'est d'abord dans des articles de presse que nous avons trouvé ces nouvelles formes, puis des recherches de celles-ci sur internet ont permis d'en trouver d'autres. Néanmoins toutes ne sont pas aussi utilisées les unes que les autres et nous avons pris en compte leur circulation dans la presse généraliste, sur internet à l'aide de divers moteurs de recherche, ainsi que celles des équivalents autochtones, quand ils existent ${ }^{2}$. L'exposé de la diversité des données trouvées, avec leur circulation dans chacune des trois langues ${ }^{3}$, précède une réflexion sur les leçons linguistiques que l’on peut tirer de ce phénomène émergent.

\section{Des selfies de parties du corps}

De même qu'il existe des « mannequins détail », auxquels on recourt pour telle ou telle partie de leur corps (pied, main, jambe, bouche, œil, etc.), de même des personnes prennent des photos de certaines parties de leurs corps et les diffusent sur internet et les réseaux sociaux. Nous les examinerons par ordre thématique (plutôt que par ordre de fréquence décroissante, qu'il est difficile d'évaluer et qui peut changer).

2 Avec Europresse pour le français de France, avec Gazeta Wyborcza - gazeta.pl, Rzeczpospolita - rp.pl et Newsweek - newsweek.pl pour le polonais et Idnes.cz, Lidovky.cz et Blesk.cz pour le tchèque. Pour internet, c'est Google qui est utilisé pour le français (de France) et le polonais, alors que pour le tchèque c'est Seznam. Les requêtes ont été faites ou actualisées fin septembre 2016.

3 Les résultats de la presse sont plus probants que ceux indiqués par les moteurs de recherche. Dans ce dernier cas, c'est moins la valeur absolue qui compte que les écarts constatés entre plusieurs mots. 


\subsection{Backselfie et belfie}

Dans backselfie c'est le dos, mais parfois surtout le bas du dos, et dans belfie ce sont les fesses qui sont l'objet du cliché proposé à l'admiration de tous ceux qui désirent le regarder. La dénomination belfie avec l'initiale $b$ - de boot « derrière, popotin » est à la fois plus et moins explicite que backselfie : sémantiquement boot est plus précis que back mais, morphologiquement, il ne serait peut-être pas évident, sans explications ou sans photos, de reconstituer boot à partir de la seule initiale $b$-. Ce cas de compocation, pour reprendre le terme créé par Cusin-Berche ([1999] 2003) reste assez opaque, du moins pour sa première partie. Nous y reviendrons en 6.1. Voici les résultats de nos enquêtes de diffusion (faites courant 2016, sur la toile (@) et dans la presse).

\begin{tabular}{|c|c|c|c|c|c|c|}
\hline \multirow{2}{*}{ backselfie } & @ fr & $@ c z$ & @pl & presse fr & presse $\mathrm{cz}$ & presse pl \\
\hline & 1320 & 1 & 2200 & $\varnothing$ & $\varnothing$ & 1 \\
\hline & @ & $@ c z$ & $@ \mathrm{pl}$ & presse fr & presse $c z$ & presse $\mathrm{pl}$ \\
\hline & 5450 & 487 & 14700 & 13 & 5 & 5 \\
\hline
\end{tabular}

Belfie est nettement plus représenté que backselfie, qui n'est attesté qu'une seule fois dans la presse, polonaise. Belfie arrive en tête de la diffusion dans la presse : $3^{\text {ème }}$ en français, $1^{\text {er }}$ en tchèque et 2 ème en polonais.

\subsection{Breastie et underboobsselfie}

Là encore le premier terme est plus vaste : "photo de sa poitrine », mais un bon nombre de sites expliquent (et montrent) qu'il s'agit surtout de poitrines féminines (du moins dans des sites non gays). Ce qui est plus opaque, c'est le fractomorphème (ou plutôt fractolexème) -ie représentant selfie. Nous reviendrons en fin d'exposé sur cet aspect morphologique. En revanche, il n'y a aucun doute dans underboobsselfie, où les deux éléments du composé sont complets, alors que les photos ainsi dénommées n'exhibent qu'une partie des seins. Ils sont vus de dessous. C'est une des rares lexies de toute la série à avoir un équivalent français, qui circule un peu : décolleté inversé, qui, s'il est parlant, contrevient à l'étymologie puisque décolleté vient de collet et de cou (col). Pour la petite histoire, c'est d'abord ou surtout en Thaillande que ce genre de clichés s'est répandu, mettant en fureur les autorités militaro-politiques du pays, qui ont décidé de sanctionner leurs auteurs, mais ces photos prises le plus souvent en gros plan 
ne permettent guère d'identifier les propriétaires des « objets » dont l'affichage public est considéré délictueux.

$\begin{array}{lcccccc} & @ \mathrm{fr} & @ \mathrm{cz} & @ \mathrm{pl} & \text { presse fr } & \text { presse cz } & \text { presse pl } \\ \text { breastie } & 679 & 12 & 110 & \varnothing & \varnothing & \varnothing \\ \text { underboobs- } & @ \mathrm{fr} & @ \mathrm{cz} & @ \mathrm{pl} & \text { presse fr } & \text { presse cz } & \text { presse pl } \\ \text { selfie } & 28000 & \varnothing & 4 & 1 & \varnothing & \varnothing\end{array}$

Si breastie a des scores faibles (679 sur Google en français, ce qui est peu par contraste avec les items pour lesquels on trouve plusieurs milliers de sites), la situation est plus contrastée pour underboobsselfie qui n'est attesté qu'une seule fois dans la presse, française, alors qu'il l'est sur beaucoup de sites internet en français, mais peu en polonais et tchèque.

\subsection{Sideboobsselfie}

Une variante de l'underboobsselfie est le sideboobsselfie qui est une photo des seins prise de côté. Cette variante est très peu représentée.

$\begin{array}{ccccccc} & @ \text { fr } & @ c z & @ p l & \text { presse fr } & \text { presse cz } & \text { presse pl } \\ \text { sideboobsselfie } & 2 & 2 & \varnothing & \varnothing & \varnothing & \varnothing\end{array}$

Le nombre très bas trouvé est le signe d'une quasi absence de diffusion de ce type de selfie qui doit rester très anecdotique.

\subsection{Topless selfie}

Dernière variante des selfies de poitrines féminines, le topless selfie, c'est-à-dire sans soutien-gorge. C'est un composé en deux mots, dont le premier est attesté depuis longtemps.

$\begin{array}{ccccccc} & @ \text { fr } & @ c z & @ p l & \text { presse fr } & \text { presse cz } & \text { presse pl } \\ \text { topless selfie } & 63200 & \varnothing & 459 & \varnothing & \varnothing & \varnothing\end{array}$

Si ce mot est absent de la presse dans les trois langues, il est bien représenté sur Google (français de France), nettement moins en polonais, et absent, ce qui ne laisse d'étonner, en tchèque. 


\subsection{Legsie / legfie/ lelfie}

Le nom des photos de ses propres jambes est clair, même hors contexte, avec le nom leg(s) complet, au pluriel ou au singulier (collectif). Mais on trouve une autre possibilité avec les deux seules premières lettres $l e-$, qui sont moins parlantes. Le fractolexème -ie du premier cas pose les mêmes problèmes d'identification que dans breastie vu auparavant, mais c'est précisément l'appartenance à une série qui permet l'identification (voir infra). La reconnaissance de selfie est plus facile dans les deux autres dénominations puisqu'on a -fie et même-elfie.

\begin{tabular}{|c|c|c|c|c|c|c|}
\hline & $@ f r$ & @cz & $@$ @l & pressefr & pressecz & pressepl \\
\hline \multirow[t]{2}{*}{ legsie } & 3390 & 320 & 2290 & 1 & $\varnothing$ & $\varnothing$ \\
\hline & @fr & @cz & $@$ pl & pressefr & pressecz & pressepl \\
\hline \multirow[t]{2}{*}{ legfie } & 593 & 7 & 1670 & $\varnothing$ & $\varnothing$ & $\varnothing$ \\
\hline & @fr & @ cz & $@ \mathrm{pl}$ & pressefr & pressecz & pressepl \\
\hline lfie & 8230 & $\varnothing$ & 708 & $\varnothing$ & $\varnothing$ & $\varnothing$ \\
\hline
\end{tabular}

Si les selfies de jambes ne sont attestés qu'une seule fois dans la presse, française (c'est peu), ils sont plus fréquents sur internet, mais lelfie est le plus présent sur la toile française alors que c'est legsie qui arrive en tête en tchèque et en polonais.

\subsection{Helfie}

Il s'agit de photos en gros plan de coiffures remarquables. Le $h$ - initial correspond à hair. Il s'agit souvent de coiffures spéciales, travaillées pour une occasion ou une autre, et qu'on veut montrer à ses amis, voire à un public plus large.

$\begin{array}{ccccccc} & @ \mathrm{fr} & @ \mathrm{cz} & @ \mathrm{pl} & \text { pressefr } & \text { pressecz } & \text { pressepl } \\ \text { helfie } & 3810 & 102 & 1280 & 7 & \varnothing & \varnothing\end{array}$

Alors qu'avec 7 occurrences dans la presse française, helfie arrive en 4 ème position dans la famille des selfies (selfie étant exclu), il est totalement absent de la presse tchèque et polonaise. 


\subsection{Nailfie}

Les ongles font également l'objet de soins de plus en plus nombreux et fréquents comme le montre la multiplication des ongleries ${ }^{4}$ dans nos villes. Il n'est dès lors pas étonnant que ces ongles décorés dans ces instituts spécialisés soient immortalisés sous forme de photos.

$\begin{array}{ccccccc} & @ f r & @ c z & @ p l & \text { pressefr } & \text { pressecz } & \text { pressepl } \\ \text { nailfie } & 931 & 2 & 32500 & \varnothing & \varnothing & 1\end{array}$

C'est en polonais que ce type de selfie apparaît surtout, beaucoup plus qu'en français et surtout qu'en tchèque où il est quasiment absent : seulement deux occurrences sur Seznam.

\subsection{Nelfie}

Le $n$ - de nelfie vaut pour nude et le mot dénomme donc des photos de soi dans le plus simple appareil, sans aucun vêtement ni voile, ou des photos au naturel, sans maquillage ni apprêt. Des photos du premier type, comme des précédents d'ailleurs, mais sans doute surtout celles-ci, peuvent être prises directement, avec ou sans canne à selfie, ou par l'intermédiaire d'une glace, ce qui permet de varier les points de vue en faisant pivoter la glace.

$\begin{array}{ccccccc} & @ f r & @ c z & @ p l & \text { pressefr } & \text { pressecz } & \text { pressepl } \\ \text { nelfie } & 6850 & 3 & 549 & \varnothing & \varnothing & \varnothing\end{array}$

Avec les moteurs de recherche pour le français, on ne trouve que les composés nude selfie et selfie nude pour des photos de nus (parfois de type pornographique) et pour des photos au naturel, sans maquillage ni retouche. Absent de la presse dans les trois langues, nelfie est attesté minimalement sur Seznam en tchèque, un peu plus en polonais et encore plus en français, mais il arrive néanmoins mal classé dans cette langue, par rapport aux autres selfies.

4 Le mot, inconnu de correcteurs orthographiques français, est encore absent du Petit Robert. Présent dans Hachette depuis au moins 2009, il est entré dans la nomenclature du Petit Larousse 2012, mais il en est ressorti dès le millésime suivant sur injonction de l'entreprise dont c'est la marque déposée. 
Nous remarquons que d'autres parties du corps, ainsi mis en morceaux, pourraient constituer l'objet de photos mises sur la toile. La série est ouverte et peut s'enrichir. Comme équivalent à l'underboobsselfie féminin, des hommes ont ainsi fait des photos, prises de dessous, de leurs testicules ou boules nommées ballfie (très peu de sites). Ce serait une pratique ancienne, antérieure à l'expansion des selfies et qui était connue sous le nom de nutscaping comme l'atteste la citation suivante: «C'est une nouvelle tendance qui s'apprête à envahir la Toile! Crée pourtant en 2007, bien avant les selfies, le Nutscaping consiste à prendre ses testicules en photo devant un sublime paysage.» (http://www.entrevue.fr/le-nutscaping-photographiez-vos-boules-devant-un-sublime-paysage/ 29 oct 2015).

Il est curieux de n'avoir pas encore trouvé de photos de son propre nombril dans cette pratique oh combien nombriliste.

\section{Selfie de sous-vêtements}

Les parties du corps objets de selfies sont, selon les cas, vêtues on non, mais certains selfies prennent précisément comme objets des sous-vêtements qui mettent en valeur ce qu'ils recouvrent.

\subsection{Bikini bridge selfie}

Le bikini bridge selfie consiste à photographier le pont que fait le maillot de bain tendu au dessus du ventre plat (ou creusé le temps du cliché).

$\begin{array}{lcccccc} & @ f r & @ c z & @ p l & \text { pressefr } & \text { pressecz } & \text { pressepl } \\ \text { bikinibridge } & 5 & 90 & 169 & \varnothing & \emptyset & 1 \\ \text { selfie } & & & & & & \end{array}$

Nous n'avons trouvé aucune page dans la presse française pour bikini bridge selfie: 3 sont dans des textes en anglais et le $4{ }^{\mathrm{ème}}$ cas se trouve dans deux mots séparés par une virgule, et de nombreuses pages indiquées ne sont pas des bikini bridges. On trouve en revanche sur internet quelques photos de bikini bridge selfie, mais elles sont plus fréquentes en tchèque et en polonais (avec aussi une attestation dans la presse de cette langue). 


\subsection{Tanga selfie}

Une autre partie de la garde-robe féminine qui peut donner naissance à une variante du selfie, c'est la culotte. Notre recherche a montré que c'est surtout la lingerie connue sous le nom de tanga (qui cache le pubis en laissant une grande partie des fesses découverte) qui a pris le dessus pour les selfies. Ce qui étonne un peu c'est l'inexistence du string selfie (le string se distingue du tanga par une ficelle plus étroite entre les fesses).

$\begin{array}{ccccccc} & @ f r & @ c z & @ p l & \text { pressefr } & \text { pressecz } & \text { pressepl } \\ \text { tangaselfie } & 8 & \varnothing & 6 & \varnothing & \varnothing & 1\end{array}$

La diffusion de tanga selfie est très faible dans les trois langues et ce type de selfie se trouve dans la fin de la liste pour ce qui est de la fréquence d'emploi, comme le suivant.

\subsection{Sexy yoga pantselfie}

Il s'agit de se prendre en photo avec une tenue, a priori confortable, permettant de s'adonner au yoga, mais cette tenue met également en valeur les charmes physiques de ceux, ou plus souvent de celles, qui le portent.

$\begin{array}{lcccccc} & @ f r & @ c z & @ p l & \text { pressefr } & \text { pressecz } & \text { pressepl } \\ \text { sexyyoga } & \varnothing & \emptyset & 1 & \varnothing & \emptyset & \emptyset \\ \text { pantselfie } & & & & & & \end{array}$

C'est le niveau le plus bas imaginable de diffusion : une seule attestation, dans une seule langue, le polonais, sur internet.

\section{Des selfies dans certaines attitudes ou activités}

À côté de ces photos de parties de soi et de vêtements qui les couvrent, se sont développées aussi des photos avec des sortes de mise en scène de soi ou de parties de soi, dans certaines attitudes, certaines activités, certains décors. 


\subsection{Bookshelfie et shelfie}

Figurent parmi les premiers types de portraits en situation, dans une série ouverte, ceux pris devant des étagères de livres ou des bibliothèques. Ces clichés valorisent le sujet (qui est aussi l'objet de la photo), comme le sont les photos de présidents de la République ou d'intellectuels devant leur bibliothèque. Le livre a ou confère encore du prestige.

Ces deux amalgames forment de véritables mots-valises ${ }^{5}$ avec la superposition d'une syllabe identique au centre [Elf]. C'est un des rares cas de mots-valises dans cette série d'amalgames, au sens large (voir infra).

\begin{tabular}{lcccccc} 
& $@ \mathrm{fr}$ & $@ \mathrm{cZ}$ & $@ \mathrm{pl}$ & pressefr & pressecz & pressepl \\
bookshelfie & 995 & 13 & 2540 & 2 & $\varnothing$ & 1 \\
& & & & & & \\
\multirow{2}{*}{ shelfie } & 27700 & 784 & 8240 & 2 & $\varnothing$ & 1
\end{tabular}

Attesté trois fois en tout dans la presse française et polonaise (mais pas tchèque), shelfie est nettement plus fréquent sur internet que bookshelfie. Le premier se classe en $4{ }^{\mathrm{ème}}$ position en français et polonais et 2 ème en tchèque, ce qui fait de shelfie un des types de selfies les mieux représentés sur internet.

\subsection{Felfie}

Loin de poser devant des bibliothèques dans des pièces fermées, certaines personnes revendiquent leur statut de fermier vivant à la campagne et diffusent des photos d'eux dans des paysages agrestes. Il faut en effet reconnaître farmer dans le $f$-initial de felfie, qui est donné comme une invention des Irlandais, et dont les premières attestations semblent dater de 2014.

$\begin{array}{ccccccc} & @ f r & @ c z & @ p l & \text { pressefr } & \text { pressecz } & \text { pressepl } \\ \text { felfie } & 628 & 52 & 2250 & 1 & 1 & 1\end{array}$

Présent une fois dans la presse de chacune des trois langues, felfie ne présente pas des scores très élevés sur internet, sauf en polonais où il arrive en 10 ème position.

5 Pour des distinctions au sein des phénomènes d'amalgamation, voir Sablayrolles (2015), Makri-Morel (2015) et Pruvost et Sablayrolles (2016). 


\subsection{Drelfie}

À l'inverse des cas précédents, il s'agit de se montrer dans une posture peu valorisante : ivre. Sans doute l'ébriété abaisse-t-elle la vigilance et altère-t-elle le jugement pour que des individus désirent s'immortaliser dans des situations qu'ils pourront regretter une fois dessoûlés. De telles photos mises sur internet ne vont pas sans risque puisque les employeurs quêtent des informations sur la toile sur les candidats à des recrutements : aussi bien l'intempérance que son affichage desservent les personnes qui diffusent ce type de photos d'eux-mêmes.

Les deux consonnes initiales $d r$ - font songer assez facilement à drunken «ivre », surtout avec les photos ainsi sous-titrées.

$\begin{array}{ccccccc} & @ f r & @ c z & @ p l & \text { pressefr } & \text { pressecz } & \text { pressepl } \\ \text { drelfie } & 679 & 22 & 766 & 6 & \emptyset & 2\end{array}$

Ce type de selfie arrive en $3^{\text {ème }}$ position dans la presse polonaise et en $5^{\mathrm{ème}}$ dans la presse française, mais il est complètement absent de la presse tchèque. La circulation sur internet est assez basse dans les trois langues.

\subsection{Smelfie}

Le ridicule ne tuant point, certains cherchent des situations où on ne se met pas à proprement parler, en valeur, en grimaçant. C'est le cas de pères (les photos sont en effet essentiellement masculines, ce qui ne laisse pas d'intriguer et en dit encore long sur le partage des tâches au sein des couples de parents) qui tordent le nez en reniflant une couche souillée de leur bébé.

$\begin{array}{ccccccc} & @ f r & @ c z & @ p l & \text { pressefr } & \text { pressecz } & \text { pressepl } \\ \text { smelfie } & 440 & 439 & 932 & \varnothing & \varnothing & \varnothing\end{array}$

C'est surtout sur internet que l'on trouve des occurrences de smelfie, avec des photos, alors qu'elles sont absentes de la presse dans les trois langues.

\subsection{Welfie}

On revient à des photos plus valorisantes de soi avec les welfie, compoqué de workout et selfie (voir 6.1). On y montre sa musculature quand on en est en train de se livrer à des exercices physiques. Les pages référencées sur Google en français de 
France ne sont pas si nombreuses, et celles où figurent des photos sont encore moins nombreuses. On n'en a relevé qu’une seule occurrence dans la presse française.

$\begin{array}{ccccccc} & @ \mathrm{fr} & @ \mathrm{cz} & @ \mathrm{pl} & \text { pressefr } & \text { pressecz } & \text { pressepl } \\ \text { welfie } & 1270 & \varnothing & 215 & 1 & \varnothing & \varnothing\end{array}$

Complètement absent du tchèque, la circulation est minimale en polonais et à peine supérieure en français : une seule occurrence dans la presse et seulement 1270 sites répertoriés. C’est peu et ce type de selfie est un des moins employés.

\subsection{Braggie}

«Le «selfie », cet autoportrait du meilleur goût réalisé bras tendu à l'aide d'un smartphone, serait-il en passe d'être supplanté par le «braggie » sur les réseaux sociaux? Le terme désigne les photos qui n'ont pour seul but que de rendre jaloux vos « amis» en posant dans des lieux censés être formidables. » (Xavier Colas, 23.11.2013 : www.terrafemina.com/.../33412-le-selfie-est-mort-vive-le-braggie.html).

$\begin{array}{ccccccc} & @ f r & @ c z & @ p l & \text { pressefr } & \text { pressecz } & \text { pressepl } \\ \text { braggie } & 584 & 8 & 421 & 1 & \varnothing & \varnothing\end{array}$

La diffusion de braggie est encore inférieure en français à celle du type de selfie précédent. Mais il y en a 8 attestations sur Seznam contre ø et celles des sites polonais ne sont pas loin du double, mais cela reste dans une fourchette basse.

\subsection{Sexfie, after sexselfie}

Des photos érotiques prises au moment des ébats sexuels, mais aussi souvent après, sont aussi diffusées sur internet, mais la presse ne s'en fait guère l'écho. Le nombre d'occurrences sur les sites polonais est supérieur à celui relevé sur les sites français.

$\begin{array}{lcccccc} & @ \text { fr } & @ \mathrm{cz} & @ \mathrm{pl} & \text { pressefr } & \text { pressecz } & \text { pressepl } \\ \text { sexfie } & 1030 & 4 & 1150 & 1 & \varnothing & \varnothing \\ & @ \mathrm{fr} & @ \mathrm{cz} & @ \mathrm{pl} & \text { pressefr } & \text { pressecz } & \text { pressepl } \\ \text { aftersexselfie } & 460 & 5 & 1660 & \varnothing & \varnothing & \varnothing\end{array}$




\subsection{Bumpie}

Alors qu'il aurait pu être classé en 1) comme partie du corps, en 2) avec des vêtements souvent adaptés, bumpie, selfie du ventre de femmes enceintes, est classé ici en 3) en tant que photo d'un état temporaire et évolutif d'un individu. Ce mot offre aussi une transition avec la $4{ }^{\text {ème }}$ partie sur le nombre des personnes figurant sur le cliché, puisque le ou les bébés à naître ne sont pas encore visibles, mais ne tarderont pas à l'être, comme le montre le type de selfie suivant (3.9).

$\begin{array}{ccccccc} & @ f r & @ c z & @ p l & \text { pressefr } & \text { pressecz } & \text { pressepl } \\ \text { bumpie } & 427 & 275 & 2650 & \varnothing & \varnothing & 2\end{array}$

Absent de la presse, bumpie figure avec des photos sur internet, mais dans de faibles proportions par rapport à d'autres types de selfies, sauf en polonais où le nombre de résultats est assez important (2650).

\subsection{Birthie}

Les accouchements et naissances font également l'objet de selfies : on fait un birthie pour immortaliser cet événement marquant et très personnel.

$\begin{array}{ccccccc} & @ f r & @ c z & @ p l & \text { pressefr } & \text { pressecz } & \text { pressepl } \\ \text { birthie } & 1520 & \varnothing & 375 & \varnothing & \varnothing & \varnothing\end{array}$

Là encore la diffusion ne se fait que sur internet, avec des photos, et elle n'est pas (encore ?) très grande.

\section{Des selfies de soi seul ou accompagné}

Si le selfie est avant tout un autoportrait photographique (egoportrait), le photographe peut n'être pas le seul sujet de la photo : il peut se trouver en compagnie. Plusieurs situations peuvent être distinguées dans les dénominations de ces photos de soi accompagné. On peut nommer la qualité des accompagnants ou adopter un point de vue quantitatif. Des objets périphériques jouent un rôle de ce point de vue. 


\subsection{La nature des accompagnants}

Nombre d'adeptes du selfie se photographient avec leur animal de compagnie préféré. On peut ainsi voir de nombreux dogselfies. Mais si on cherche sur internet on trouve aussi d'autres dénominations comprenant en première partie le nom de l'animal, comme cat selfie. Néanmoins, ils semblent moins circuler dans la presse généraliste et nous n'en avons pas trouvé spontanément, alors que les chiffres sur internet donnent des résultats équivalents pour les chats et les chiens : 3760 catselfie ont été répertoriés sur les pages en français de France de Google le 29 janvier 2016 face à 3000 dogselfie. Le nombre a doublé en quelques mois pour atteindre 7610 et 7560 en septembre. Ces deux mots circulent également sur internet en polonais et en tchèque, mais seul cat selfie apparaît dans la presse, française et tchèque, et on constate aussi une sorte de dévoiement de l'utilisation de selfie dans la mesure où, sur nombre de ces photos, l'auteur de la photographie n'apparaît pas. Nous y reviendrons.

$\begin{array}{ccccccc} & @ \mathrm{fr} & @ \mathrm{cz} & @ \mathrm{pl} & \text { pressefr } & \text { pressecz } & \text { pressepl } \\ \text { catselfie } & 7610 & 7 & 3210 & 2 & 1 & \emptyset \\ & @ \mathrm{fr} & @ \mathrm{cz} & @ \mathrm{pl} & \text { pressefr } & \text { pressecz } & \text { pressepl } \\ \text { dogselfie } & 7560 & 13 & 4410 & \varnothing & \varnothing & \emptyset\end{array}$

Il est aussi courant de s'immortaliser avec des amis lors de rencontres chez soi, en extérieur, à l'occasion de festivités diverses (anniversaires, enterrements de vie de garçon ou de jeune fille, etc.). Bref, les situations ne manquent pas où on peut s'adonner au friendsie, et faire partager à tous ceux qui le veulent sur la toile des moments inoubliables. Mais le nombre de pages consacrées à la série «Friends» dans les moteurs de recherches ne permet pas de trouver facilement de clichés ainsi nommés ni de connaître la circulation sur internet de ce mot, absent par ailleurs des archives de presse consultées.

Et certains, si contents de leur bonheur, tiennent à le faire savoir largement en diffusant des relfies. "Contraction de "selfie" et de "relationship", le relfie désigne un autoportrait au smartphone, mettant en scène plusieurs personnes qui s'aiment ou, tout du moins, font mine de s'apprécier. » (http://www.airofmelty.fr/le-relfie-la-nouvelle-tendance-inspiree-du-selfie-sur-les-reseaux-sociaux-a307263.html).

$\begin{array}{ccccccc} & @ f r & @ c z & @ p l & \text { pressefr } & \text { pressecz } & \text { pressepl } \\ \text { relfie } & 673 & \varnothing & 421 & 1 & \varnothing & 1\end{array}$


Avec une seule occurrence de presse dans deux langues, relfie apparaît, pour la diffusion, en $5^{\text {ème }}$ et dernière position en polonais et $9^{\text {ème }}$ et dernière en français. Sa circulation sur la toile est également assez restreinte.

Il y a aussi des cas de ce qui est nommé photobomb selfie, avec l'intrusion (volontaire ?) d'une personne inconnue sur la photo. À l'inverse, on peut faire un selfie en compagnie de quelqu'un qui ne le souhaite pas, en particulier si celui-ci est assoupi. C'est la mésaventure qui est arrivée à Jean-Marie Le Pen, qui a gagné le procès qu'il a intenté à celui qui s'était pris en photo avec lui, alors qu'il s'était endormi en avion.

$\begin{array}{lcccccc} & @ \mathrm{fr} & @ \mathrm{cz} & @ \mathrm{pl} & \text { pressefr } & \text { pressecz } & \text { pressepl } \\ \text { photobomb- } & 605 & 19 & 340 & \varnothing & \varnothing & 1 \\ \text { selfie } & & & & & & \end{array}$

On ne relève qu'une seule attestation dans la presse, polonaise, et des occurrences peu nombreuses sur internet.

\subsection{Le nombre des accompagnants}

Avec une seule personne autre que soi-même on réalise un twofie, et à partir de deux et au-delà, un usie ou ussie (une photo de «nous »). C'est alors le nombre des personnes et non la qualité de celles-ci qui est pris en compte. L'autre ou les autres personnes ne sont pas nécessairement des proches, mais ça peut être des personnes célèbres auprès desquelles on veut figurer pour attester qu'on les a rencontrées. Ce peut être aussi des rencontres occasionnelles, sans lendemain : groupe de travail, photos de vacances, etc.

$\begin{array}{ccccccc} & @ \mathrm{fr} & @ \mathrm{cz} & @ \mathrm{pl} & \text { pressefr } & \text { pressecz } & \text { pressepl } \\ \text { twofie } & 419 & 3 & 142 & \varnothing & \varnothing & \varnothing \\ & @ \mathrm{fr} & @ \mathrm{cc} & @ \mathrm{pl} & \text { pressefr } & \text { pressecz } & \text { pressepl } \\ & & & & \varnothing & \varnothing & 1\end{array}$

Ces deux mots sont quasiment absents de la presse puisqu'on ne trouve qu'une seule occurrence de twofie dans la presse, polonaise. La circulation de ce mot n'est pas très importante sur la toile et celle de us(s)ie n'est pas mesurable car les requêtes donnent les résultats de Russie. 


\subsection{Des périphériques / accessoires}

Peu après l'avènement du selfie, a été inventé un objet, le selfie stick, qui permet de se prendre avec plus de recul, en éloignant de soi l'appareil photo qui se trouve à l'extrémité d'une canne ou perche. On peut donc figurer dans un environnement, un paysage plus vaste (pour attester qu'on a été à tel ou tel endroit et faire envie à ses amis) ou avec un groupe de personnes plus ou moins important. Le selfie stick peut être simple, mais il est plus souvent télescopique. Son emploi est parfois interdit, comme c'est le cas dans nombre de musées par exemple.

C'est un des rares mots de la série à avoir un équivalent, et ce dans les trois langues. Il y en a même plusieurs : bras à selfie, canne à selfie ou perche à selfie pour le français.

$\begin{array}{ccccccc} & @ \mathrm{fr} & @ \mathrm{cz} & @ \mathrm{pl} & \text { pressefr } & \text { pressecz } & \text { pressepl } \\ \text { selfie stick } & 111000 & 132375 & 233000 & 20 & 5 & 23\end{array}$

$\begin{array}{llllll}0 \mathrm{fr} & @ \mathrm{cz} @ \mathrm{pl} \text { pressefr } & \text { pressecz } & \text { pressepl }\end{array}$

bâton à selfie

ø $510795 \quad 33700$

$\emptyset$

112

2

kijek selfie

selfie tyč

$\begin{array}{lcccccc} & @ \mathrm{fr} & @ \mathrm{C} z & @ \mathrm{pl} & \text { pressefr } & \text { pressecz } & \text { pressepl } \\ \text { perche à selfie } & 110000 & 242076 & 5 & 146 & 46 & \emptyset \\ \text { tyczka do selfie } & & & & & & \\ \text { selfie tyčka } & & & & & & \end{array}$

$\begin{array}{lcccccc} & @ f r & @ c z & @ p l & \text { pressefr } & \text { pressecz } & \text { pressepl } \\ \text { bras à selfie } & 679 & 804542 & 560 & 5 & \varnothing & 1 \\ \text { rączka do selfie } & & & & & & \\ \text { selfie držák } & & & & & & \end{array}$

Le nom de cet instrument est sans conteste un de ceux de cette famille qui s'est le plus diffusé : il arrive en première position dans la presse polonaise et tchèque et en seconde dans la presse française. Les scores relevés sur internet sont également très élevés. Il est à noter que les équivalents autochtones (on constate un foisonnement néologique dans les trois langues) sont plus utilisés en français et en tchèque alors que le polonais privilégie l'emprunt. 
Le mot dronie est ambigu ; c'est une " photo de soi avec un drone ", mais avec le double sens de avec, " accompagnement » : l'individu et le drone figurent tous deux sur le cliché (dans ce cas il pourrait être classé avec 4.1), ou « recours à un instrument » : c'est, de fait, aussi et surtout, le fait de se prendre en photo soi-même à l'aide d'un drone, ce qui augmente considérablement le recul, par rapport à un simple selfie stick.

$\begin{array}{ccccccc} & @ f r & @ c z & @ p l & \text { pressefr } & \text { pressecz } & \text { pressepl } \\ \text { dronie } & 2260 & 610 & 153 & 22 & \varnothing & \emptyset\end{array}$

Alors que c'est le mot de cette famille qui est le plus présent dans la presse française, il est complètement absent de la presse polonaise et tchèque, ce qui est curieux. Il est vrai que l'apparition de ce mot semble assez récente et il faut peutêtre lui donner le temps de franchir les frontières. La circulation sur internet en français ne correspond pas à sa diffusion dans la presse : elle reste modeste. Dans le cas de la langue polonaise la recherche semble plus compliquée du fait que la forme graphique dronie est une forme déclinée (au datif) du substantif dron.

\section{Bilan des données et de leur circulation dans les trois langues}

Si on relève quasiment autant d'items de cette famille en français (19) et en polonais (17), le tchèque en présente beaucoup moins (7), mais cela tient sans doute à des différences dans les sources utilisées. En revanche le nombre d'occurrences des emprunts relevés dans la presse et de leurs équivalents rapproche le français (219) et le tchèque (173) alors que le polonais se singularise avec seulement 47. Il $\mathrm{y}$ a là un paradoxe puisque le tchèque a beaucoup d'occurrences pour un faible nombre d'items (ratio de 24,7) et que c'est exactement l'inverse en polonais (ratio de 2,8), mais ce paradoxe vient du grand nombre de selfie stick et équivalents en tchèque (163). Dans cette langue, comme dans les deux autres, on relève un grand nombre d'occurrences uniques dans la presse : il y a en 7 sur 19 en français (37\%), 2 sur 7 en tchèque ( $28,5 \%$ ) et 10 sur 17 en polonais (58,9\%). Ce qui est très fréquent dans les trois langues, et le plus fréquent dans deux d'entre elles, c'est le nom de l'instrument qui permet de prendre les selfies avec une distance plus grande que la seule longueur du bras : le selfie stick. On en compte 20 en français, 5 en tchèque et 23 en polonais avec, respectivement, 156, 158 et 3 équivalents 
autochtones. Ces chiffres montrent le contraste entre le polonais, qui privilégie l'emprunt aux équivalents autochtones, et les deux autres langues, où la situation est inverse. Un autre contraste est patent : alors qu'en français dronie est le selfie qui apparaît en première position dans la presse avec 22 occurrences, il est complètement absent de la presse polonaise et tchèque. Ce qui apparaît partagé dans la presse des trois langues est assez rare. C'est le cas de belfie qui est bien représenté dans la presse des trois langues : en $1^{\text {ère }}$ position en tchèque, en $2^{\text {nde }}$ en polonais et en 3 ème en français. Felfie est attestée une fois dans chacune des trois langues

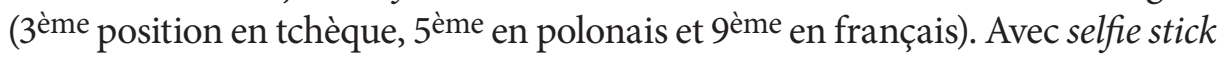
cela ne fait que trois items présents dans la presse des trois langues. Il est vrai que ce phénomène s'épanouit plus volontiers sur la toile où le nombre d'occurrences relevées est beaucoup plus élevé. On constate des écarts numériques importants entre le tchèque d'une part, avec le moteur de recherche très utilisé dans ce pays, Seznam, et le français et le polonais d'autre part, pour lesquels on a utilisé Google. C'est probablement la différence entre les moteurs de recherche qui explique ces disparités numériques. Mais on constate aussi une grande disparité dans l'ordre de fréquence des items, sauf pour selfie stick, toujours en tête. Les sites français ont plus de selfies de poitrines que de fesses alors que c'est l'inverse en tchèque et polonais. Les sites polonais sont nombreux pour nailfie (2 ${ }^{\mathrm{ème}}$ position) alors qu'ils le sont beaucoup moins en français et surtout en tchèque. Les selfies devant des étagères à livres (shelfie) sont bien représentés dans les trois langues. Le prestige du livre fonctionne encore. L'amour des animaux de compagnie aussi, du moins en français et en tchèque.

\section{Aspects morpho-sémantiques}

D’un point de vue linguistique et plus précisément morpho-sémantique, plusieurs constatations peuvent être faites à partir de ces données foisonnantes.

\subsection{Modes de formation : mot-valisation, compocation, fractocomposition}

Pour ce qui est des modes de formation, on relève des composés en deux ou trois mots graphiques (bikini bridge selfie) ou un seul (underboobsselfie), avec parfois les deux possibilités (cat selfie et catselfie). Il n'y a en revanche que très peu de cas de mots-valises du type shelfie et boookshelfie dans lesquels un segment ho- 
mophone [Elf] vaut deux fois, par superposition syllabique, une fois à la fin du premier mot (shelf, bookshelf) et la seconde comme partie de la syllabe initiale du deuxième mot (selfie), avec juste la légère différence phonique qui oppose la consonne sifflante et la consonne chuintante. Aucun cas de factorisation au sens de Julie Makri-Morel (2015) n'est relevé. Il s'agit des amalgames dans lesquels la ou les séquences phoniques homophones ne sont pas à l'intersection des deux mots. Les matrices les plus utilisées dans la création des mots de cette famille de selfies sont la compocation et la fractocomposition. Dans le premier cas, il y a troncation des deux mots fondus en un seul. Chacun des mots est représenté par un fragment de lui-même qui vaut pour le tout (v. Cusin-Berche, [1999] 2003). Belfie, helfie, lelfie, nelfie, etc. sont des compoqués dans lesquels -elfie vaut pour selfie et les consonnes initiales $b$-, $h$-, l-, n-valent pour boot, hair, leg, nude. Dans la fractocomposition, un seul des mots est tronqué, l'autre étant complet. Dans birthie, legsie, nailfie, etc., les mots initiaux birth, legs, nail sont complets, mais le mot selfie est représenté par une partie de lui-même -ie ou-fie.

\subsection{Une série de fractolexèmes}

Ces cas de compocation et de fractocomposition aboutissent à l'émergence de toute une série de fractolexèmes. Si le mot selfie est parfois, mais rarement, présent en entier dans un certain nombre de mots relevés dans cette étude ( $\log$ selfie, underboobsselfie, etc.), on relève en effet des cas plus nombreux où selfie est représenté par une partie du mot, plus ou moins large et reconnaissable. Quand selfie se réduit à -elfie avec la seule perte du s-initial, comme dans drelfie ou relfie, le mot est assez reconnaissable et le sens n'est pas difficile à trouver, même si on n'a pas encore rencontré le mot. La reconnaissabilité diminue mais reste encore assez grande pour -lfie avec la perte des deux premières lettres se-, comme dans nailfie, où le -l-peut appartenir au début du second mot ou à la fin du premier. La consécution des lettres - lf- suivie de -ie renvoie assez facilement à selfie. En revanche la suppression de la troisième lettre - $l$-abouti à -fie que l'aphérèse rend moins directement identifiable à selfie. Dans ce cas, comme dans le suivant où selfie se réduit à -ie (legsie...), c'est l'existence d'une floraison de dénominations pour divers types de selfies qui guide l'interprétation, mais ça demande néanmoins parfois un effort important et certaines dénominations constituent des sortes de devinettes. C'est d'ailleurs parfois présenté ainsi dans les journaux et hebdomadaires ("Savez-vous ce qu'est un Xie?»). Seules les photos et les explications permettent d'assurer le lecteur de ce dont il s'agit. Remarquons de surcroît que la rareté des mots anglais se terminant par-ie, en dehors d'une série 
de monosyllabes tels die, lie, tie...) et de noms de tribus (hippie, yuppie, yuccie...) et de quelques autres comme junkie, groupie, etc. facilite l'identification de selfie dans les dénominations des nouveaux types de selfies se terminant en -ie.

\subsection{Des cas de synonymie}

Nous avons vu en passant des cas curieux de synonymie, liés précisément au fait de l'existence de plusieurs fractomorphèmes (ou plutôt fractolexèmes) pour chacun des deux éléments entrant dans la composition de la dénomination de la photo. Ce fut le cas de legsie, legfie et lelfie. Nous ne nous y appesantissons pas plus et passons à des cas de polysémie puis d'homonymie, facilités aussi par les troncations et la présence de fractolexèmes.

\subsection{Des cas de polysémie}

Dans nelfie, $n$-vaut pour nude, dans deux acceptions « nu » ou « non maquillé », qui ont comme point commun sémantique de renvoyer à quelque chose ou quelqu'un de naturel. On parle bien de naturistes pour les nudistes, avec l'absence de vêtement et l'état de nature. Mais le naturel s'oppose aussi à l'artificiel, par absence de fard, de maquillage. C'est donc une même idée qui se réalise sous des réalités différentes. Aussi est-il sans doute préférable de voir ici de la polysémie plutôt que de l'homonymie.

\subsection{Des cas d'homonymie}

L'émergence de fractolexèmes, de mots réduits à une partie d'eux-mêmes qui les représente en entier, ne va sans ambiguité quand le segment de l'élément autre que selfie est réduit au point de se résumer à un seul phonème (une seule lettre à l'écrit), qui peut correspondre à plusieurs mots. Ainsi si relfie s'est développé récemment avec le sens de « relationship selfie, au sens de relation amoureuse [...], les médias tiennent à rappeler que le relfie existe depuis déjà de nombreuses années, mais pour des significations différentes. Ainsi, successivement, le relfie a pu désigner un selfie pris avec un membre de la famille royale (Royal Sselfie $<$ sic $>$ ) ou le cliché d'une main portant une bague de fiançailles ou une alliance (Ring Selfie) ». Le site d'où est tirée cette citation ajoute que «son sens pourrait bien changer de nouveau ».

Welfie est surtout attesté pour des selfies de quelqu'un qui s'entraîne physiquement et qui met ainsi en valeur ses qualités sportives, mais on trouve 
quelques cas de ce mot pour des selfies avec une mise en scène où la personne se prend en photo dans une cave ou un magasin de vins et spiritueux, avec des casiers de bouteilles de vin comme arrière plan. Le $w$-vaut alors pour wine alors qu'il valait pour workout dans le premier cas, plus fréquent.

\subsection{Des cas d'hyperonymie?}

On peut s'interroger sur la relation sémantique qui semble exister parfois entre un terme générique (backselfie) et un terme plus spécifique (belfie) dans une relation de type hyperonymique. Si certaines photos de backselfie sont bien des photos de dos, dans un grand nombre de cas il s'agit du bas du dos, et plus précisément des fesses, ce que dénomme proprement belfie. Il ne semble pas qu' il faille voir dans ce recours à un hyperonyme moins précis une volonté d'atténuation ou d'euphémisme pour des pratiques assez exhibitionnistes et des photos suggestives.

\subsection{Des cas de conversion}

Tous les emprunts ou allogénismes et les équivalents autochtones, dans les trois langues, sont des noms, mais est apparue en français par conversion (et détournement d'une citation célèbre de Descartes) une forme verbale, selfier, dans « Je selfie, donc je suis », titre d'un livre récent d'Elsa Godart, paru en mai 2016 chez Albin Michel. Dans «Tous selfie », titre d'un autre livre paru à peu près simultanément, aux éditions nouvelles François Bourin, sous la plume d'une universitaire, Pauline Escande-Gauquié, selfie est probablement devenu adjectif, également par conversion. Ces deux cas montrent l'implantation du mot en français.

\section{Quelques leçons linguistiques autres que morpho-sémantiques}

\subsection{Une grande productivité}

On ne peut que constater un incontestable effet d'entraînement qui consiste à inventer, lancer, diffuser de nouveaux types de selfies, avec plus ou moins de succès, mais plus par défi ludique que pour implanter durablement ces innovations. Nul ne sait cependant ce qui va être repris ou pas, et l'ampleur de la diffusion, et cela 
dans le monde entier. On remarque que cette effervescence créative n'est pas toujours durable et qu'elle ne pénètre que modérément dans les médias généralistes. On remarque aussi des trous, des lacunes pour des parties du corps ou certaines attitudes. Ainsi nous n'avons pas (encore) relevé de selfie du nombril ou des parties du corps tatoués ou avec des piercings, etc. En revanche a été trouvé plus récemment, le 10 octobre 2016, un nouveau type de selfie qui se présente comme un défi : un selfie où on se montre en train de frapper dans ses mains. Mais ce qui est remarquable dans cette dernière invention, c'est qu'elle n'est pas nommée.

\subsection{Rareté des équivalents autochtones}

On constate que la plupart des mots de la famille ne connaissent pas d'équivalents autochtones à la notable exception du français décolleté inversé pour underboobsselfie, et, pour les trois langues de perche, bras ou canne à selfie et selfie tyč, kijek selfie, tyczka do selfie, selfie tyčka, rączka do selfie, selfie držák pour selfie stick. On trouve aussi couplie, synonyme de relfie, hybride associant le mot français couple et le fractolexème anglais -ie valant pour selfie.

Cette absence, ou du moins rareté, d'équivalents est sans doute liée au patron phonétique et la fin en -ie qui fonde l'unité de cette famille de mots. Il existe cependant, nous a-t-on signalé, des selflous, forme atypique à plusieurs égards. Outre l'absence du -ie final, c'est un mot-valise (dont on a vu qu' ils étaient rares), hybride de surcroît associant la lexie anglaise self- avec la lexie française flou, avec le segment consonantique commun [fl] $-f l$ -

\subsection{Des innovations linguistiques : emprunts et/ou allogénismes}

Cette multiplication des créations s'est accompagnée d'innovations linguistiques avec l'émergence d'une famille de fractolexèmes. Ce qui est remarquable, c'est leur nombre (on a relevé pas moins de quatre formes différentes), le maximum de ce qui est imaginable, et le fait qu'au moins une des formes, -ie dans usie par exemple) n'est guère interprétable que par l'existence du groupe. Mais il y a aussi l'aspect devinette qui est mis en action. En l'occurrence usfie, tout à fait pensable, aurait été moins opaque que usie, sans être nécessairement complètement limpide.

La circulation internationale des mots de cette famille, ou du moins d'une grande partie d'entre eux, conduit à s'interroger sur leur statut d'emprunts ou d’allogénismes. Ce n'est pas parce que la quasi totalité de ces mots comportent des éléments anglais qu'il sont nés en anglais et ont été empruntés à cette langue. Ils peuvent très bien avoir été fabriqués dans d'autres pays et d'autres 
langues avec le recours à des formants anglais. Mais c'est souvent indécidable car il est difficile de trouver à coup sûr la première attestation, et même si on y parvenait, il pourrait y avoir une recréation dans une autre langue que celle où le mot est apparu en premier (que ce soit l'anglais ou une autre langue). Il s'agit de cas d'internationalismes, quelle que soit la langue où le mot est créé et la / les langues par lesquelles l'innovation transite.

\subsection{Un aspect ludique}

La recherche de la nouveauté et de la concision ainsi que le désir de créer des énigmes ont conduit à produire des homonymes. Mais certains sont si rares qu'on n'en trouve qu'une ou de rares traces. La série est ouverte, et il y a une sorte d'émulation ludique à inventer de nouveaux types de selfies, bizarres et inattendus, avec des dénominations en partie énigmatiques. Beaucoup sont en effet présentés comme des devinettes dans les magazines, friands de nouveautés susceptibles d'amuser et séduire leur lectorat. Apparaissent maintenant des défis, comme les très récents selfies pris en se frappant les mains.

\subsection{Une fréquente démotivation}

Il faut noter encore que le succès de cette famille de mots fait que certains d'entre eux sont employés alors même qu'il ne s'agit pas à proprement parler de selfies puisque le photographié n'est pas nécessairement le photographieur. Dans ces cas, il s'agit du mode traditionnel de la prise de photos (sauf pour des photos prises avec des retardateurs). Et il y a aussi des exemples plus complexes de photographies de personnes prises en train de se photographier : ce ne sont pas, étymologiquement, des selfies mais des photographies de selfies. C'est l'autophotographieur photographié, mais la mode fait que tous ces clichés sont dénommés du nom selfie ou d'un de ses fractolexèmes, qu'il s'agisse d'un véritable egoportrait (écrit aussi égoportrait ou ego-portrait) ou non.

\section{Autres conclusions (partielles)}

Il faut noter encore que la multiplication des selfies disponibles sur internet dit quelque chose de la société dans laquelle nous vivons, avec un penchant accru à l'égotisme (on se regarde le nombril : voilà qui pourrait, comme nous l'avons 
déjà indiqué, fournir l'objet d'un nouveau type de selfie) et à une sorte d'exhibitionnisme. Mais ces selfies montrent de surcroît une répartition inégale par sexe, ce dont les auteurs de ces lignes ne sont pas responsables, comme on l'a reproché à l'un d'entre eux! À part drelfie, shelfie, smelfie et welfie (dans le sens cave à vin) où des hommes apparaissent souvent, ce sont quasi exclusivement, ou du moins très majoritairement, des selfies féminins qui sont présents sur la toile.

Le selfie ne renvoie peut-être pas nécessairement à celui qui accomplit l'acte de se photographier (avec réflexivité), mais à celui qui se met en valeur. Mais ceci n'est pas nouveau comme en attestent les photos devant un massif de fleurs, devant un monument, en adoptant une pause, etc. qu'on n'appelait pas selfies, mais parfois « photos japonaises». Il reste que les développements technologiques avec les appareils photos numériques où on peut se voir en train de se photographier, et l'internet où on peut mettre à la disposition d'un grand nombre de gens, connus ou inconnus, ses photos ont complètement changé les pratiques antérieures.

Ce n'est sans doute pas un hasard si deux livres de sociologues / philosophes sont parus quasi simultanément : il s'agit d'un fait de société dont l'émergence est récente (2013), a été soudaine et connaît toujours un grand engouement, avec des formes renouvelées. Et c'est sans doute lié en grande partie au soin que l'on prend de soi dans des instituts de beauté, des centres de fitness, au développement du quantified self, qui sont des marques de l'importance accrue que les gens portent à eux-mêmes, du moins dans les sociétés occidentalisées, mais peut-être pas seulement.

\section{Références bibliographiques}

Cusin-Berche Fabienne, [1999] 2003, « Des mots qui bougent : le lexique en mouvement $»$ in Les mots et leurs contextes, Paris, Presses de la Sorbonne Nouvelle, p. 29-49.

Makri-Morel Julie, 2015, « Mots-valises : quand les segments communs se font la malle $»$ in Neologica 9, p. 63-82.

Pruvost Jean et Sablayrolles Jean-François, [2003] 2016, 3 ème éd., Les néologismes, Que sais-je ?, Paris, PUF.

Sablayrolles Jean-François, 2015, «Quelques remarques sur une typologie des néologismes: Amalgamation ou téléscopage : un processus aux productions variées (mots valises, détournements...) et un tableau hiérarchisé des matrices » in Neologia das linguas romanicas, Ieda Maria Alves et Eliane Simôes Pereira éd., Sâo Paulo, Humanitas, p. 187-218. 